\title{
Aortic dimensions in patients with bicuspid and tricuspid aortic valves
}

\author{
Veronica Jackson, $\mathrm{MD},{ }^{\mathrm{a}}$ Christian Olsson, $\mathrm{MD}, \mathrm{PhD},{ }^{\mathrm{a}}$ Per Eriksson, $\mathrm{PhD},{ }^{\mathrm{b}}$ and \\ Anders Franco-Cereceda, $\mathrm{MD}, \mathrm{PhD}^{\mathrm{a}}$
}

\begin{abstract}
Objective: Whether patients with bicuspid aortic valves are susceptible not only to ascending aortic dilatation but also to dilatation of the remaining aorta is uncertain. This study evaluated the possible correlation between ascending aortic dilatation and dilatation of the remaining aorta in patients with bicuspid and tricuspid aortic valves, respectively.
\end{abstract}

Methods: The entire aortas of 97 patients (bicuspid in 62, tricuspid in 35) undergoing replacement of the ascending aorta because of ascending aortic pathology ( 78 with and 19 without concurrent valve pathology) were preoperatively evaluated using computed tomography scanning.

Results: Aortic dimensions distal to the ascending aorta were smaller in patients with bicuspid aortic valves than in patients with tricuspid aortic valves $(P<.001)$ : proximal arch: $3.20 \pm 0.60 \mathrm{~cm} / 3.80 \pm 0.68 \mathrm{~cm}$; distal arch: $2.90 \pm 0.60 \mathrm{~cm} / 3.40 \pm 0.50 \mathrm{~cm}$; isthmus, $2.95 \pm 0.60 \mathrm{~cm} / 3.50 \pm 0.60 \mathrm{~cm}$; descending aorta: $2.70 \pm 0.50 \mathrm{~cm} /$ $3.20 \pm 0.65 \mathrm{~cm}$; suprarenal aorta: $2.30 \pm 0.40 \mathrm{~cm} / 2.70 \pm 0.43 \mathrm{~cm}$; infrarenal aorta: $1.90 \pm 0.48 \mathrm{~cm} / 2.20 \pm 0.35$ $\mathrm{cm}$, respectively. Concomitant pathologic dilatation of the descending aorta was more common in patients with tricuspid aortic valves $(37 \%)$ than in patients with bicuspid aortic valves $(5 \%)(P<.001)$. Neither valvular pathology nor bicuspid aortic valve phenotype influenced the aortic dimensions in the bicuspid group $(P>.2)$.

Conclusions: Patients with bicuspid aortic valves and dilatation of the ascending aorta consistently had smaller dimensions of the remaining aorta compared with patients with tricuspid aortic valves. Concomitant dilatation of the descending aorta was predominantly found in patients with tricuspid aortic valves. (J Thorac Cardiovasc Surg 2013;146:605-10)

Bicuspid aortic valve (BAV) is a common cardiac malformation occurring in $0.5 \%$ to $2 \%$ of the population. Patients with BAV present with valvular pathology (ie, stenosis or regurgitation) earlier in life than patients with tricuspid aortic valve (TAV) and are predisposed to dilatation and dissection of the ascending aorta. Thus, BAV entails an increased risk of morbidity and mortality.

The underlying mechanisms of ascending aortic dilatation in patients with BAV are still a matter of debate. The susceptibility to development of ascending aortic pathology found in patients with BAV is thought to be the result of

\footnotetext{
From the Cardiothoracic Surgery Unit at the Department of Molecular Medicine and Surgery, ${ }^{a}$ Karolinska Institutet and Karolinska University Hospital, Stockholm, Sweden; and Atherosclerosis Research Unit at the Center for Molecular Medicine at the Department of Medicine, ${ }^{\mathrm{b}}$ Karolinska Institutet, Stockholm, Sweden.

Funding: This study was supported by the Mats Kleberg foundation, the Swedish Research Council (12660), the Stockholm County Council, the Swedish Heart-Lung foundation, the Tornspiran foundation, the European Commission (FAD, HealthF2-2008-200647), and a donation by Fredrik Lundberg.

Disclosures: Authors have nothing to disclose with regard to commercial support.

Presented at the Aortic Symposium, New York, New York, April 26-27, 2012.

Received for publication March 20, 2012; revisions received May 29, 2012; accepted for publication July 25, 2012; available ahead of print Sept 17, 2012.

Address for reprints: Veronica Jackson, MD, Cardiothoracic Surgery Unit, N3:03,

Karolinska University Hospital, 17176 Stockholm, Sweden (E-mail: veronica.

jackson@karolinska.se).

$0022-5223 / \$ 36.00$

Copyright (c) 2013 by The American Association for Thoracic Surgery

http://dx.doi.org/10.1016/j.jtcvs.2012.07.039
}

a congenital defect involving the ascending aortic wall or the result of altered blood flow patterns due to BAV morphology. ${ }^{2,3}$ There is still disagreement on the optimal treatment strategy and long-term follow-up regimen of patients with BAV with aortic dilatation. ${ }^{4,5}$ There are data suggesting partly similar molecular mechanisms in ascending aortic aneurysm formation in patients with BAV, as in patients with Marfan syndrome. ${ }^{6}$ Patients with Marfan syndrome are at risk of developing aortic pathology of the distal aorta, ${ }^{7}$ which raises the question if the same is true for patients with BAV.

Reports of evaluation of the entire aorta in patients with BAV are scarce. Therefore, the aim of this study was to investigate whether there is a relation between ascending aortic dilatation in patients with BAV who underwent ascending aortic surgery and concomitant dilatation of the remaining aorta and a further comparison of BAV and TAV in this respect.

\section{MATERIALS AND METHODS \\ Patients}

The study population consisted of 97 patients (BAV in 62, TAV in 35) undergoing elective heart surgery between February 13, 2007, and February 12, 2012. These patients were studied in the setting of the Advanced Study of Aortic Pathology, a prospective, single-center, observational cohort study of patients with aortic valve or ascending aortic disease with an indication for elective surgery $(\mathrm{n}=500) .{ }^{8}$ The present retrospective 


\section{Abbreviations and Acronyms \\ $\mathrm{BAV}=$ bicuspid aortic valve \\ $\mathrm{BSA}=$ body surface area \\ $\mathrm{CT}=$ computed tomography \\ $\mathrm{TAV}=$ tricuspid aortic valve}

analysis included patients undergoing surgery for dilatation of the ascending aorta $(n=97)$ in combination with aortic valve stenosis $(n=21)$, aortic valve regurgitation $(\mathrm{n}=57)$, or a normally functioning valve $(\mathrm{n}=19)$, all of whom had undergone preoperative examination of the entire aorta by computed tomography (CT). Patients with Marfan syndrome were excluded. Definite valve phenotype (TAV or BAV) was determined intraoperatively. The patient characteristics are summarized in Table 1 .

\section{Surgical Procedure}

All patients underwent operation in a standardized fashion as previously described. ${ }^{8}$ A supracoronary graft was used alone $(\mathrm{n}=18)$ or in combination with a mechanical valve prosthesis $(\mathrm{n}=5)$, a biological valve prosthesis $(\mathrm{n}=16)$, aortic valve plasty $(\mathrm{n}=5)$, a biological composite graft (Medtronic Freestyle, Medtronic Inc, Minneapolis, Minn) $(\mathrm{n}=16)$, or arch surgery $(\mathrm{n}=2)$. A mechanical valved graft (St Jude Medical, Inc, St Paul, Minn) $(n=14)$ or a biological composite graft (Medtronic Freestyle) $(\mathrm{n}=5)$ alone was used in 19 patients, and reimplantation ad modum David $(n=12)$ alone or in combination with aortic valve plasty $(n=4)$ was used in 16 patients.

\section{Computed Tomography Examination}

The local hospital protocol for CT examinations was followed. Eighty milliliters of contrast medium ( $300 \mathrm{mg}$ iodine per $\mathrm{mL}$, Omnipaque, GE Healthcare, Ltd, Little Chalfont, UK) was administrated into an antecubital vein at a flow rate of $4 \mathrm{~mL} / \mathrm{s}$ using a power injector. The delay time for the images was determined by bolus-tracking. All CT images were reviewed by a single experienced external radiologist blinded to aortic valve morphology and subsequent surgical procedure. Maximal outer wall measurements were obtained in the axial plane in the following sections of the aorta: aortic annulus, sinus of Valsalva, sinotubular junction, ascending aorta, proximal arch, distal arch, aortic isthmus, descending aorta, suprarenal aorta, and infrarenal aorta (Figure 1 and Table 2).

\section{Definitions}

The evaluation and definition of valvular morphology (ie, TAV or BAV, including BAV phenotype) and aortic valve pathology (ie, aortic valve stenosis or aortic valve regurgitation) used in this study have been described. ${ }^{8}$ The indication for ascending aortic surgery with or without concomitant valve replacement/reconstruction was based on current surgical guidelines, ${ }^{9}$ but it was ultimately at the surgeon's discretion. The descending aorta and the infrarenal aorta were considered dilated at the diameters suggested and described by Wanhainen and colleagues ${ }^{10}$ (descending aorta $\geq$ $3.7 \mathrm{~cm}$ in men and $\geq 3.3 \mathrm{~cm}$ in women; infrarenal aorta $\geq 3.0 \mathrm{~cm}$ in men and $\geq 2.7 \mathrm{~cm}$ in women).

\section{Ethical Approval}

The study was approved by the regional Human Research Ethics Committee. Oral and written consent were obtained from all patients.

\section{Statistical Analysis}

Continuous variables are given as mean with standard deviation or median with interquartile range as appropriate, and categorical variables are presented as frequency and percentage. Mann-Whitney $U$ test or Student $t$
TABLE 1. Patient characteristics according to valvular morphology

\begin{tabular}{lccc}
\hline & $\begin{array}{c}\text { TAV } \\
(\mathbf{n}=\mathbf{3 5})\end{array}$ & $\begin{array}{c}\text { BAV } \\
(\mathbf{n}=\mathbf{6 2})\end{array}$ & $\boldsymbol{P}$ value \\
\hline Age (y) & $63 \pm 12$ & $59 \pm 13$ & .084 \\
Gender, n (M/F) & $22 / 13$ & $42 / 20$ & $>.2$ \\
BSA & $2.00 \pm 0.27$ & $1.96 \pm 0.22$ & $>.2$ \\
BMI & $27 \pm 3$ & $26 \pm 4$ & $>.2$ \\
Hypertension, ${ }^{*} \mathrm{n}$ & 24 & 36 & $>.2$ \\
Diabetes, ${ }^{\text {n }}$ & 0 & 2 & $>.2$ \\
CVL (preoperative), n & 2 & 2 & $>.2$ \\
COPD, ${ }^{*}$ n & 0 & 1 & $>.2$ \\
Normal valve, n $(\%)$ & $6(17)$ & $13(21)$ & $>.2$ \\
Aortic stenosis, n $(\%)$ & 0 & $21(34)$ & $<.001$ \\
Aortic regurgitation, n $(\%)$ & $29(83)$ & $28(45)$ & $<.001$ \\
\hline
\end{tabular}

Values are given as mean \pm standard deviation, frequency (n), or percentage (\%). $T A V$, Tricuspid aortic valve; $B A V$, bicuspid aortic valve; $B S A$, body surface area; $B M I$, body mass index; $C V L$, cerebrovascular lesion; $C O P D$, chronic obstructive pulmonary disease. *As defined by ongoing medical treatment.

test was used for comparisons between continuous data as appropriate. The Fisher exact test was used for comparison of categorical data. For comparisons of the aortic diameters in patients with BAV shown in Tables 3 and 4, Kruskall-Wallis 1-way analysis of variance was used. In Table 3, the between-group factor was "valve pathology" with 3 levels (normal valve, aortic stenosis, and aortic regurgitation). In Table 4, the between-group factor was "BAV phenotype" with 3 levels (fusion of the right and left coronary cusps, fusion of the right and noncoronary cusps, and true BAV). Analysis of covariance was also used in comparisons between patients with BAV and patients with TAV to adjust for age, gender, body surface area (BSA), and comorbidities. Statistical analyses were performed using StatView software (v 5.0.1; SAS Institute Inc, Cary, NC).

\section{RESULTS}

Of the 97 patients, all undergoing heart surgery because of dilatation of the ascending aorta, 64\% had BAV $(\mathrm{n}=62)$ and $36 \%$ had TAV $(\mathrm{n}=35)$. Patients with BAV and TAV were comparable with regard to preoperative characteristics. However, the distribution of aortic valve pathology differed between the groups. Aortic valve stenosis was found only in patients with BAV, whereas aortic valve regurgitation was more common in patients with TAV (Table 1). In the BAV group, 48 patients (77\%) had fusion of the right and left coronary cusps, 8 patients $(13 \%)$ had fusion of the right and noncoronary cusps, and 6 patients $(10 \%)$ had a true BAV.

\section{Dimensions of the Aortic Arch, Descending Aorta, and Abdominal Aorta}

No differences in aortic dimensions at the level of the annulus, the sinus of Valsalva, the sinotubular junction, or the ascending aorta were found when comparing the BAV and TAV groups. The dimensions of the proximal arch, distal arch, aortic isthmus, descending aorta, suprarenal aorta, and infrarenal aorta were consistently smaller in patients with BAV in comparison with patients with TAV (Table 2). 


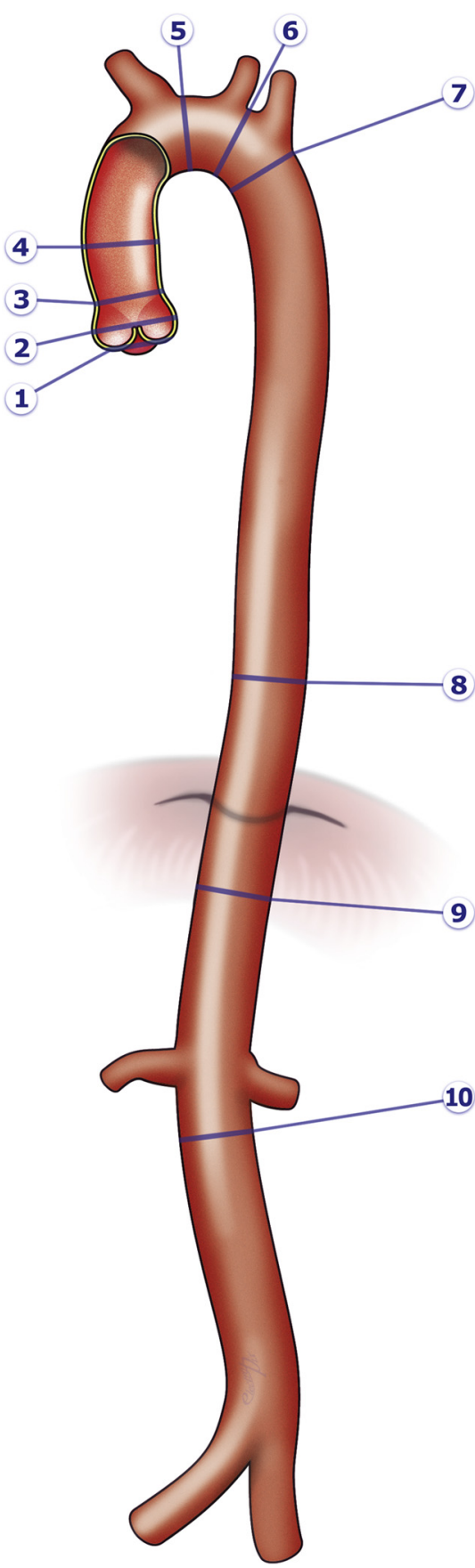

FIGURE 1. Schematic illustration of the aortic measure points: $1=$ annulus; $2=$ sinus of Valsalva; $3=$ sinotubular junction; $4=$ ascending aorta (ie, maximal aortic width distal to the sinotubular junction and proximal to the innominate artery); $5=$ proximal aortic arch (ie, maximal aortic width distal to the innominate artery and proximal to the left common carotid artery); $6=$ distal aortic arch (ie, maximal aortic width distal to the left common carotid artery and proximal to the left subclavian artery); $7=$ aortic isthmus; $8=$ descending aorta (ie, maximal aortic width distal to the aortic isthmus and proximal to the diaphragm); $9=$ suprarenal aorta (ie, maximal aortic width distal to the coeliac trunk and proximal to the superior mesenteric artery); $10=$ infrarenal aorta (ie, maximal aortic width distal to the most caudal renal artery).
TABLE 2. Aortic dimensions according to valvular morphology

\begin{tabular}{lllc}
\hline & \multicolumn{1}{c}{$\begin{array}{c}\text { TAV } \\
(\mathbf{n}=\mathbf{3 5})\end{array}$} & \multicolumn{1}{c}{$\begin{array}{c}\text { BAV } \\
(\mathbf{n}=\mathbf{6 2})\end{array}$} & $\boldsymbol{P}$ value \\
\hline Annulus & $2.40(0.40)$ & $2.50(0.40)$ & $>.2$ \\
& {$[1.19(0.23)]$} & {$[1.27(0.19)]$} & .054 \\
Sinus of Valsalva & $4.30(1.75)$ & $4.10(0.80)$ & $>.2$ \\
& {$[2.14(0.63)]$} & {$[2.16(0.40)]$} & $>.2$ \\
Sinotubular junction & $4.30(1.18)$ & $3.85(0.90)$ & .057 \\
& {$[2.06(0.48)]$} & {$[1.97(0.41)]$} & .085 \\
Ascending aorta & $5.30(0.78)$ & $5.20(0.40)$ & .108 \\
& {$[2.76(0.91)]$} & {$[2.60(0.43)]$} & $>.2$ \\
Proximal arch & $3.80(0.68)$ & $3.20(0.60)$ & $<.001$ \\
& {$[1.92(0.64)]$} & {$[1.68(0.43)]$} & .014 \\
Distal arch & $3.40(0.50)$ & $2.90(0.60)$ & $<.001$ \\
Aortic isthmus & {$[1.77(0.50)]$} & {$[1.52(0.25)]$} & $<.001$ \\
& $3.50(0.60)$ & $2.95(0.60)$ & $<.001$ \\
Descending aorta & {$[1.79(0.54)]$} & {$[1.53(0.30)]$} & $<.001$ \\
& $3.20(0.65)$ & $2.70(0.50)$ & $<.001$ \\
Suprarenal aorta & {$[1.59(0.66)]$} & {$[1.36(0.26)]$} & $<.001$ \\
& $2.70(0.43)$ & $2.30(0.40)$ & $<.001$ \\
Infrarenal aorta & {$[1.33(0.52)]$} & {$[1.15(0.19)]$} & .002 \\
& $2.20(0.35)$ & $1.90(0.48)$ & $<.001$ \\
& {$[1.11(0.32)]$} & {$[0.92(0.21)]$} & .022 \\
\hline
\end{tabular}

Values are given as median with interquartile range, in centimeters for diameters, and as centimeter per square meter for diameter indexed to BSA (values within square brackets). $T A V$, Tricuspid aortic valve; $B A V$, bicuspid aortic valve.

\section{Aortic Dimensions in Relation to Valvular Pathology and Bicuspid Aortic Valve Phenotype}

When analyzing the aortic dimensions according to valvular pathology (ie, normal function, stenosis, or regurgitation), no differences were found within the BAV group. At the level of the annulus, sinus of Valsalva, and sinotubular junction, patients with TAV and a regurgitant valve had larger crude aortic dimensions compared with patients with normal valve function. When BSA was taken into consideration, patients with TAV with valve regurgitation still had larger aortic dimensions at the level of the sinus of Valsalva, sinotubular junction, and distal arch, but not at the level of the annulus (Table 3).

No differences in aortic dimensions within the BAV group were detected on the basis of valve phenotype, that is, fusion of the right and left coronary cusps, fusion of the right and noncoronary cusps, or true BAV (Table 4).

\section{Concomitant Dilatation of the Descendning Aorta and/or Infrarenal Aorta}

Dilatation of the descending aorta was found in 13 patients $(37 \%)$ with TAV and 3 patients $(5 \%)$ with BAV. Dilatation of the infrarenal aorta was found in 3 patients $(9 \%)$ with TAV and 5 patients $(8 \%)$ with BAV. Dilatation of the descending aorta and the infrarenal aorta was found in 2 patients with TAV and 1 patient with BAV, respectively. Thus, concomitant dilatation of the descending aorta was more common in the TAV group than in the BAV group 
TABLE 3. Aortic dimensions according to valve pathology

\begin{tabular}{|c|c|c|c|c|c|c|c|}
\hline & \multicolumn{4}{|c|}{ BAV } & \multicolumn{3}{|c|}{ TAV } \\
\hline & $\begin{array}{l}\text { Valve stenosis } \\
\quad(\mathbf{n}=\mathbf{2 1})\end{array}$ & $\begin{array}{l}\text { Valve regurgitation } \\
\quad(\mathbf{n}=\mathbf{2 8})\end{array}$ & $\begin{array}{l}\text { Normal valve } \\
\quad(n=13)\end{array}$ & $P$ value & $\begin{array}{l}\text { Valve regurgitation } \\
\qquad(\mathbf{n}=\mathbf{2 9})\end{array}$ & $\begin{array}{l}\text { Normal valve } \\
\quad(n=6)\end{array}$ & $P$ value \\
\hline \multirow[t]{2}{*}{ Annulus } & $2.60(0.45)$ & $2.50(0.33)$ & $2.40(0.20)$ & $>.2$ & $2.50(0.45)$ & $2.20(0.30)$ & .040 \\
\hline & {$[1.31(0.23)]$} & {$[1.20(0.17)]$} & {$[1.26(0.12)]$} & .097 & {$[1.22(0.23)]$} & {$[1.16(0.10)]$} & $>.2$ \\
\hline \multirow[t]{2}{*}{ Sinus of Valsalva } & $4.00(0.53)$ & $4.20(0.75)$ & $4.10(1.10)$ & $>.2$ & $4.60(1.50)$ & $3.45(0.80)$ & .007 \\
\hline & {$[2.08(0.33)]$} & {$[2.17(0.30)]$} & {$[2.23(0.78)]$} & $>.2$ & {$[2.20(0.61)]$} & {$[1.89(0.06)]$} & .006 \\
\hline \multirow[t]{2}{*}{ Sinotubular junction } & $3.70(0.70)$ & $3.90(0.90)$ & $3.90(1.00)$ & $>.2$ & $4.40(0.88)$ & $3.25(0.60)$ & .003 \\
\hline & [1.93 (0.30)] & {$[2.00(0.38)]$} & [2.01 (0.46)] & $>.2$ & {$[2.10(0.42)]$} & {$[1.83(0.20)]$} & .002 \\
\hline \multirow[t]{2}{*}{ Ascending aorta } & $5.10(0.58)$ & $5.20(0.40)$ & $5.30(0.28)$ & .153 & $5.30(0.98)$ & $5.70(0.70)$ & $>.2$ \\
\hline & {$[2.59(0.37)]$} & {$[2.60(0.49)]$} & {$[2.76(0.31)]$} & $>.2$ & {$[2.52(0.98)]$} & {$[2.87(0.59)]$} & $>.2$ \\
\hline \multirow[t]{2}{*}{ Proximal arch } & $3.50(0.53)$ & $3.20(0.53)$ & $3.25(0.45)$ & .093 & $3.80(0.70)$ & $3.85(1.10)$ & $>.2$ \\
\hline & {$[1.75(0.44)]$} & {$[1.60(0.38)]$} & [1.77 (0.29)] & .066 & {$[1.87(0.65)]$} & {$[2.09(0.27)]$} & .111 \\
\hline \multirow[t]{2}{*}{ Distal arch } & $3.00(0.70)$ & $2.90(0.55)$ & $2.90(0.38)$ & $>.2$ & $3.30(0.43)$ & $3.60(1.30)$ & .115 \\
\hline & {$[1.57(0.29)]$} & {$[1.50(0.33)]$} & {$[1.53(0.13)]$} & $>.2$ & {$[1.74(0.44)]$} & {$[1.93(0.60)]$} & .034 \\
\hline \multirow[t]{2}{*}{ Aortic isthmus } & $2.90(0.63)$ & $2.95(0.70)$ & $3.00(0.50)$ & $>.2$ & $3.50(0.70)$ & $3.60(1.40)$ & $>.2$ \\
\hline & {$[1.52(0.33)]$} & {$[1.49(0.38)]$} & {$[1.54(0.21)]$} & $>.2$ & {$[1.77(0.53)]$} & {$[2.01(0.46)]$} & .115 \\
\hline \multirow[t]{2}{*}{ Descending aorta } & $2.60(0.60)$ & $2.80(0.55)$ & $2.70(0.33)$ & $>.2$ & $3.20(0.63)$ & $3.50(1.00)$ & .063 \\
\hline & {$[1.37(0.25)]$} & {$[1.34(0.29)]$} & {$[1.38(0.19)]$} & $>.2$ & {$[1.55(0.60)]$} & {$[1.92(0.56)]$} & .054 \\
\hline \multirow[t]{2}{*}{ Suprarenal aorta } & $2.30(0.43)$ & $2.30(0.40)$ & $2.30(0.30)$ & $>.2$ & $2.70(0.40)$ & $2.80(0.70)$ & .102 \\
\hline & {$[1.19(0.22)]$} & [1.12(0.19)] & {$[1.16(0.12)]$} & $>.2$ & {$[1.27(0.48)]$} & [1.58 (0.39)] & .056 \\
\hline \multirow[t]{2}{*}{ Infrarenal aorta } & $1.80(0.35)$ & $1.90(0.45)$ & $1.85(0.30)$ & $>.2$ & $2.20(0.40)$ & $2.35(0.50)$ & $>.2$ \\
\hline & {$[0.87(0.11)]$} & {$[0.95(0.32)]$} & {$[0.91(0.10)]$} & $>.2$ & {$[1.10(0.34)]$} & {$[1.14(0.15)]$} & $>.2$ \\
\hline
\end{tabular}

Values are given as median with interquartile range, in centimeters for diameters, and as centimeter per square meter for diameter indexed to BSA (values within square brackets). $B A V$, Bicuspid aortic valve; $T A V$, tricuspid aortic valve.

(37\% vs $5 \% ; P<.001)$, whereas dilatation of the infrarenal aorta alone and in combination with dilatation of the

TABLE 4. Aortic dimensions according to bicuspid aortic valve phenotype

\begin{tabular}{lllll}
\hline & \multicolumn{1}{c}{$\mathbf{R L}$} & \multicolumn{1}{c}{$\mathbf{R N}$} & \multicolumn{1}{c}{ True BAV } \\
$(\mathbf{n}=\mathbf{4 8})$ & \multicolumn{1}{c}{$(\mathbf{n}=\mathbf{8})$} & \multicolumn{1}{c}{$(\mathbf{n}=\mathbf{6})$} & $\boldsymbol{P}$ value \\
\hline Annulus & $2.50(0.38)$ & $2.55(0.30)$ & $2.55(0.40)$ & $>.2$ \\
& {$[1.27(0.21)]$} & {$[1.27(0.15)]$} & {$[1.31(0.21)]$} & $>.2$ \\
Sinus of Valsalva & $4.10(0.70)$ & $4.10(0.40)$ & $4.75(1.30)$ & $>.2$ \\
& {$[2.13(0.40)]$} & {$[2.08(0.39)]$} & {$[2.23(0.44)]$} & $>.2$ \\
Sinotubular junction & $3.75(0.90)$ & $4.00(0.75)$ & $4.10(0.60)$ & $>.2$ \\
& {$[1.96(0.42)]$} & {$[1.98(0.34)]$} & {$[2.06(0.23)]$} & $>.2$ \\
Ascending aorta & $5.20(0.40)$ & $5.25(0.75)$ & $4.95(0.50)$ & $>.2$ \\
& {$[2.61(0.40)]$} & {$[2.61(0.37)]$} & {$[2.53(0.56)]$} & $>.2$ \\
Proximal arch & $3.20(0.60)$ & $3.50(0.43)$ & $3.10(1.30)$ & .136 \\
& {$[1.64(0.42)]$} & {$[1.75(0.23)]$} & {$[1.71(0.61)]$} & $>.2$ \\
Distal arch & $2.90(0.55)$ & $3.25(0.40)$ & $2.90(0.80)$ & $>.2$ \\
& {$[1.50(0.29)]$} & {$[1.63(0.20)]$} & {$[1.54(0.23)]$} & $>.2$ \\
Aortic isthmus & $2.95(0.60)$ & $3.25(0.75)$ & $2.85(0.60)$ & $>.2$ \\
& {$[1.53(0.32)]$} & {$[1.55(0.16)]$} & {$[1.46(0.16)]$} & $>.2$ \\
Descending aorta & $2.70(0.60)$ & $2.85(0.50)$ & $2.65(0.20)$ & $>.2$ \\
& {$[1.36(0.26)]$} & {$[1.38(0.11)]$} & {$[1.31(0.18)]$} & $>.2$ \\
Suprarenal aorta & $2.30(0.43)$ & $2.45(0.30)$ & $2.30(0.20)$ & $>.2$ \\
& {$[1.15(0.20)]$} & {$[1.16(0.07)]$} & {$[0.15(0.23)]$} & $>.2$ \\
Infrarenal aorta & $1.90(0.50)$ & $1.80(0.43)$ & $1.85(0.25)$ & $>.2$ \\
& {$[0.91(0.31)]$} & {$[0.94(0.10)]$} & {$[0.86(0.06)]$} & $>.2$ \\
\hline Values are given as median with interquartile range, in centimeters for diameters, and \\
as centimeter per square meter for diameter indexed to BSA (values within square \\
brackets). RL, Right and left coronary cusp fusion; $R N$, right and noncoronary cusp \\
fusion; BAV, bicuspid aortic valve. & & & \\
& & & &
\end{tabular}

descending aorta was equally common in both groups $(P$ $>.2$ in both instances).

\section{DISCUSSION}

In this study of 97 patients with dilated ascending aorta undergoing ascending aortic surgery because of ascending aortic pathology with or without concomitant valvular pathology, patients with BAV consistently had smaller arch, descending, suprarenal, and infrarenal aortic dimensions than patients with TAV. Furthermore, patients with TAV had concomitant pathologic dilatation of the descending aorta to a greater extent than patients with BAV. There were no differences in aortic root and ascending aortic dimensions between the BAV and TAV groups. In addition, neither valvular pathology nor BAV phenotype influenced the aortic dimensions in the BAV group, which is in accord with previous findings of the lack of relation between BAV phenotype and aortic root morphology. ${ }^{8}$

Analysis of covariance was used to adjust for age, gender, BSA, and certain comorbidities, and the differences in aortic dimensions found between BAV and TAV groups could not be assigned to these factors. There were no detectable differences in aortic dimensions related to valvular pathology when analyzing the BAV group alone, and only minor differences in aortic root dimensions were found in the TAV group in relation to a normally functioning or regurgitant valve (Tables 3 and 4). 
The finding that patients with BAV with ascending aortic dilatation had smaller dimensions of the remaining aorta than patients with TAV is in agreement with previous echocardiographic evaluation of individuals with BAV who did not undergo surgery. Cecconi and co-workers ${ }^{11}$ retrospectively evaluated, using transthoracic echocardiography, the dimensions of the entire aorta in 162 patients with BAV without valvular pathology and stratified them according to age. They found a prevalence of $28 \%$ to $77 \%$ of ascending aortic dilatation in the different age strata but could not find any relation between BAV and dilatation of the descending or abdominal aorta. Our data could not confirm the findings by Fazel and colleagues ${ }^{12}$; in their study, $28 \%$ of patients with BAV with dilated ascending aorta also had concomitant dilatation of the transverse arch. However, it should be pointed out that a direct comparison is difficult to make because of differences in measure points and method of analysis. Alegret and colleagues ${ }^{13}$ have reported different profiles of aortic diameter in relation to valvular morphology based on CT examinations of the aorta in 71 patients with dilated aortic root primarily identified by echocardiography. The authors pointed out a high prevalence of concomitant abdominal aortic aneurysms in patients aged more than 60 years with TAV but not BAV. Conversely, Larsson and colleagues ${ }^{14}$ reported that patients with abdominal aortic aneurysms had a high frequency of thoracic aortic aneurysm mainly located in the descending part of the thoracic aorta, although the relative distribution of BAV and TAV was unknown in their study. In our cohort of patients, all of whom underwent operation for a dilated ascending aorta, concomitant pathologic dilatation of the descending aorta was more common in patients with TAV than in patients with BAV (37\% vs 5\%). Taken together, these findings support that BAV-associated dilatation of the aorta is present in the aortic root and/or ascending aorta but not in the descending or abdominal aorta. ${ }^{11}$ In contrast, dilatation of the aorta in patients with TAV can be present in all aortic segments.

The underlying pathologic mechanisms of aortic aneurysm formation are multifaceted. Aortic aneurysms, regardless of location (ie, ascending, descending, or abdominal), are associated with medial degeneration, which is characterized by loss of elastic fibers and smooth muscle cells. Inflammation and atherosclerosis are highly associated with abdominal aortic aneurysm, which is to some extent a factor in descending aortic aneurysm formation but not a major contributor to aneurysm formation of the ascending aorta. ${ }^{15}$ To complicate the matter, the histopathologic and genetic features of descending and abdominal aortic aneurysms differ. ${ }^{16}$ Moreover, mediators of medial degeneration ${ }^{6,17}$ and activation of immune response ${ }^{18}$ in aneurysm formation of the ascending aorta differ between patients with BAV and patients with TAV. Thus, ascending aortic aneurysm formation in patients with $\mathrm{TAV}$, in contrast to patients with BAV, is partly influenced by inflammation and immune response, ${ }^{18}$ which implies that the underlying molecular mechanisms of aneurysm formation of the ascending and descending aortas are similar in patients with TAV but differ in patients with BAV.

The relative contribution of ascending aortic blood flow changes to BAV-associated aortic dilatation has been addressed by numerous studies with partly conflicting results. A morphologically normal BAV may cause turbulent flow resulting in aortic dilatation, ${ }^{19}$ and BAV phenotype per se has been shown to evoke abnormal helical flow patterns in the ascending aorta. ${ }^{20}$ This may support the findings of aortic aneurysm in patients with BAV with functionally normal valves. $^{21,22}$ However, progressive aortic dilatation begins early in childhood in patients with $\mathrm{BAV},{ }^{23}$ and a clear correlation of valve pathology and aortic dimensions has not been shown. ${ }^{24,25}$ Moreover, the morphology of the aortic root and ascending aorta is similar regardless of valvular morphology (ie, BAV or TAV), as well as BAV phenotype. ${ }^{8}$ In addition, the lack of detectable differences in dimensions of the aortic root and ascending aorta between patients with TAV and patients with BAV, and within the BAV group alone, in the present cohort does not support altered hemodynamics as the primary cause of aortic dilatation in patients with BAV. The underlying mechanisms of aneurysm formation need to be further evaluated, preferably taking valve morphology (BAV/TAV), localization of the aneurysm (ascending, arch, descending, abdominal), and aortic valve pathology (stenosis, regurgitation) into account.

\section{Study Limitations}

The present study group represents a limited cohort accepted for surgery because of ascending aortic dilatation and with variations in valve phenotype and pathology; therefore, the findings may not be applicable to a general, healthy population with BAV. As previously shown, ${ }^{8}$ aortic valve stenosis is rare in patients with TAV with a dilated ascending aorta, and this should be taken into account when interpreting the results. However, the patients represent a defined group subjected to a standardized preoperative examination interpreted by an external and blinded radiologist.

\section{CONCLUSIONS}

In patients undergoing surgery for ascending aortic dilatation, patients with BAV consistently showed smaller dimensions of the remaining aorta compared with patients with TAV. The pattern of cusp fusion and valve function did not influence the aortic dimensions in patients with BAV. Concomitant dilatation of the descending aorta was predominantly found in patients with TAV. Therefore, the present results may be used to reassure patients with BAV of a small likelihood of a pathologically dilated remaining aorta when undergoing surgery of the ascending aorta. 
The authors thank Elisabeth Berg (Medical Statistics Unit, Karolinska Institutet) for assistance in the statistical review of this study.

\section{References}

1. Siu SC, Silversides CK. Bicuspid aortic valve disease. J Am Coll Cardiol. 2010; 55:2789-800.

2. Loscalzo ML, Goh DL, Loeys B, Kent KC, Spevak PJ, Dietz HC. Familial thoracic aortic dilation and bicommissural aortic valve: a prospective analysis of natural history and inheritance. Am J Med Genet A. 2007;143A: 1960-7.

3. Nathan DP, Xu C, Plappert T, Desjardins B, Gorman JH 3rd, Bavaria JE, et al. Increased ascending aortic wall stress in patients with bicuspid aortic valves. Ann Thorac Surg. 2011;92:1384-90.

4. Svensson LG, Kim KH, Blackstone EH, Rajeswaran J, Gillinov AM, Mihaljevic T, et al. Bicuspid aortic valve surgery with proactive ascending aorta repair. J Thorac Cardiovasc Surg. 2011;142:622-9.

5. Park CB, Greason KL, Suri RM, Michelena HI, Schaff HV, Sundt TM 3rd. Fate of nonreplaced sinuses of valsalva in bicuspid aortic valve disease. $J$ Thorac Cardiovasc Surg. 2011;142:278-84.

6. Fedak PW, de Sa MP, Verma S, Nili N, Kazemian P, Butany J, et al. Vascular matrix remodeling in patients with bicuspid aortic valve malformations: implications for aortic dilatation. J Thorac Cardiovasc Surg. 2003; 126:797-806.

7. von Kodolitsch Y, Robinson PN. Marfan syndrome: an update of genetics, medical and surgical management. Heart. 2007;93:755-60.

8. Jackson V, Petrini J, Caidahl K, Eriksson MJ, Liska J, Eriksson P, et al. Bicuspid aortic valve leaflet morphology in relation to aortic root morphology: a study of 300 patients undergoing open-heart surgery. Eur J Cardiothorac Surg. 2011;40: e118-24.

9. Bonow RO, Carabello BA, Chatterjee K, de Leon AC Jr, Faxon DP, Freed MD, et al. ACC/AHA 2006 guidelines for the management of patients with valvular heart disease: a report of the American College Of Cardiology/American Heart Association Task Force on Practice Guidelines (writing committee to revise the 1998 Guidelines for the Management of Patients With Valvular Heart Disease) developed in collaboration with the Society of Cardiovascular Anesthesiologists endorsed by the Society for Cardiovascular Angiography and Interventions and the Society of Thoracic Surgeons. J Am Coll Cardiol. 2006;48:e1-148.

10. Wanhainen A, Themudo R, Ahlstrom H, Lind L, Johansson L. Thoracic and abdominal aortic dimension in 70-year-old men and women-a population-based whole-body magnetic resonance imaging (MRI) study. J Vasc Surg. 2008;47: 504-12.

11. Cecconi M, Manfrin M, Moraca A, Zanoli R, Colonna PL, Bettuzzi MG, et al. Aortic dimensions in patients with bicuspid aortic valve without significant valve dysfunction. Am J Cardiol. 2005;95:292-4.
12. Fazel SS, Mallidi HR, Lee RS, Sheehan MP, Liang D, Fleischman D, et al. The aortopathy of bicuspid aortic valve disease has distinctive patterns and usually involves the transverse aortic arch. J Thorac Cardiovasc Surg. 2008; 135:901-7.

13. Alegret JM, Calvo N, Ligero C, Palomares R, Millá L, Martín-Paredero V, et al. Dilated aortic root is related to a global aortic dilating diathesis. J Vasc Surg. 2010;52:867-72.

14. Larsson E, Vishnevskaya L, Kalin B, Granath F, Swedenborg J, Hultgren R. High frequency of thoracic aneurysms in patients with abdominal aortic aneurysms. Ann Surg. 2011;253:180-4.

15. Isselbacher EM. Thoracic and abdominal aortic aneurysms. Circulation. 2005; 111:816-28.

16. Absi TS, Sundt TM 3rd, Tung WS, Moon M, Lee JK, Damiano RR Jr, et al. Altered patterns of gene expression distinguishing ascending aortic aneurysms from abdominal aortic aneurysms: complementary DNA expression profiling in the molecular characterization of aortic disease. J Thorac Cardiovasc Surg. 2003; 126:344-57.

17. Jackson V, Olsson T, Kurtovic S, Folkersen L, Paloschi V, Wagsater D, et al. Matrix metalloproteinase 14 and 19 expression is associated with thoracic aortic aneurysms. J Thorac Cardiovasc Surg. 2012;144:459-66.

18. Folkersen L, Wagsater D, Paloschi V, Jackson V, Petrini J, Kurtovic S, et al. Unraveling the divergent gene expression profiles in bicuspid and tricuspid aortic valve patients with thoracic aortic dilatation: the ASAP study. Mol Med. 2011;17: 1365-73.

19. Robicsek F, Thubrikar MJ, Cook JW, Fowler B. The congenitally bicuspid aortic valve: How does it function? Why does it fail? Ann Thorac Surg. 2004;77: 177-85.

20. Hope MD, Hope TA, Meadows AK, Ordovas KG, Urbania TH, Alley MT, et al. Bicuspid aortic valve: four-dimensional $\mathrm{mr}$ evaluation of ascending aortic systolic flow patterns. Radiology. 2010;255:53-61.

21. Hahn RT, Roman MJ, Mogtader AH, Devereux RB. Association of aortic dilation with regurgitant, stenotic and functionally normal bicuspid aortic valves. J Am Coll Cardiol. 1992;19:283-8

22. Nistri S, Sorbo MD, Marin M, Palisi M, Scognamiglio R, Thiene G. Aortic root dilatation in young men with normally functioning bicuspid aortic valves. Heart. 1999;82:19-22.

23. Beroukhim RS, Kruzick TL, Taylor AL, Gao D, Yetman AT. Progression of aortic dilation in children with a functionally normal bicuspid aortic valve. Am J Cardiol. 2006;98:828-30.

24. Della Corte A, Bancone C, Quarto C, Dialetto G, Covino FE, Scardone M, et al. Predictors of ascending aortic dilatation with bicuspid aortic valve: a wide spectrum of disease expression. Eur J Cardiothorac Surg. 2007;31: 397-404.

25. Linhartova K, Beranek V, Sefrna F, Hanisova I, Sterbakova G, Peskova M. Aortic stenosis severity is not a risk factor for poststenotic dilatation of the ascending aorta. Circ J. 2007; 71:84-8. 